\title{
Presentation II: Precision Medicine in migraine management
}

Theodora Barkoula ${ }^{1, *}$

${ }^{1}$ Neurological Clinic, General Oncology Hospital, "Agioi Anargyroi", Athens, Greece. * dora.bark@gmail.com

Migraine is a common disorder that negatively affects a significant percentage of patients, with corresponding social and economic costs. It is a neurovascular disease whose pathophysiological mechanisms are still being examined. While cortical spreading depression (CSD) and dysfunction of the trigeminovascular system appear to play a key role in the onset of migraine aura and pain, the study of human genome has shown the involvement of several genes and neuropeptides in its pathogenesis. New data make it necessary to search for targeted methods in the diagnosis and treatment of the disease, as current therapeutic approaches in many cases show little effectiveness and significant side effects.

Nowadays, the rapid development of genetics opens new horizons in the approach of migraine through the prism of Medical Precision. Precision Medicine (PM) is a new approach to the treatment and prevention of the disease, which combines the individualized approach of the patient with the design and implementation of appropriate treatment. It differs radically from the "one size fits all" approach, leading to a better understanding of the pathophysiological mechanisms of the disease and the development of innovative diagnostic and treatment options. It combines the expression of genome and the correlation of science of Biochemistry with the comorbidity of the patient, in order to achieve the optimal clinical result.

The purpose of this essay is to present the current therapeutic approaches for migraine treatment and to investigate their applicability, according to the new data of Medical Precision. The investigation and identification of new genetic, epigenetic, biochemical and other biomarkers will enable the patient to be better approached, more targeted treatment and reduced disease's management costs.

\section{Keywords:}

Migraine; Precision medicine; Biomarkers; Genome; Neuropeptides; Genetics; Therapy 